\title{
Safety of chidamide plus rituximab in elderly patients with relapsed or refractory B-cell lymphoma in China: a multicenter, single-arm, phase II study
}

\author{
Xinrui Chen ${ }^{1,2}$, Huaqing Wang ${ }^{1,2}$, Xiuhua Sun ${ }^{3}$, Liping Su ${ }^{4}$, Fengting Liu ${ }^{1,2}$, Ke Zhao ${ }^{1,2}$, Liye Xu ${ }^{3}$, \\ Shaohua $\mathrm{Wu}^{4}$, Teng Song ${ }^{1,2}$ \\ ${ }^{1}$ Department of Oncology, Tianjin Union Medical Center, Nankai University, Tianjin, China; ${ }^{2}$ The Institute of Translational Medicine, Tianjin \\ Union Medical Center, Nankai University, Tianjin, China; ${ }^{3}$ Department of Oncology, Second Hospital of Dalian Medical University, Dalian, \\ Liaoning, China; ${ }^{4}$ Department of Hematology, Shanxi Provincial Cancer Hospital, Taiyuan, China \\ Contributions: (I) Conception and design: H Wang, F Liu, X Chen, X Sun, L Su; (II) Administrative support: H Wang, F Liu, X Chen, X Sun, L Su; (III) \\ Provision of study materials or patients: X Chen, K Zhao, L Xu, S Wu, T Song; (IV) Collection and assembly of data: X Chen, K Zhao, L Xu, S Wu, \\ T Song; (V) Data analysis and interpretation: X Chen; (VI) Manuscript writing: All authors; (VII) Final approval of manuscript: All authors. \\ Correspondence to: Huaqing Wang; Fengting Liu. 190 Jieyuan Street, Hongqiao District, Tianjin, China. Email: huaqingw@163.com; liufengting@tjmuch.com.
}

Background: Patients over 65 years old with diffuse large B-cell lymphoma (DLBCL) or follicular lymphoma (FL) relapse or being refractory to rituximab-associated chemotherapy have limited treatment options. Chidamide has the ability to enhance the sensitivity of rituximab-resistant tumors in vivo has been confirmed. We aimed to assess the activity and safety profile of chidamide plus rituximab in elderly Chinese patients with recurrent or refractory B-cell lymphoma.

Methods: In this prospective, single-arm phase II trial, we enrolled patients from three hospitals in China with histopathological diagnoses of DLBCL and FL who had relapsed or were refractory to previous lines of rituximab-associated chemotherapy. Patients were given chidamide (10 mg on days 1-6 and 8-14) and rituximab $\left(375 \mathrm{mg} / \mathrm{m}^{2}\right.$ on day 7). The treatments were repeated every 21 days. The primary endpoint was the objective response rate (ORR). The secondary endpoints included the disease control rate (DCR), progression-free survival (PFS), overall survival (OS), and safety.

Results: Thirteen patients were enrolled and commenced treatment between November 12, 2018, and December 24, 2020. As of March 20, 2021, two patients (15.4\%) were still receiving treatment. The median follow-up was 13.4 months. The ORR was $40 \%$ for the DLBCL cohort $(n=10)$, and $100 \%$ for the FL cohort $(n=3)$. DLBCL patients had a median PFS (mPFS) of 2.6 months (0.9-31.2 months) and a median OS (mOS) of 16.7 months (2.3-13.6 months). Neither mPFS nor mOS was reached in the FL cohort. The most frequent treatment-related adverse events (TRAEs) were leukopenia (38.5\%), neutropenia (30.8\%), lymphopenia (30.8\%), thrombocytopenia (30.8\%), fatigue (38.5\%), and hyperuricemia (30.8\%).

Conclusions: Chidamide plus rituximab is clinically effective with an acceptable toxicity profile in elderly patients over 65 years old with relapsed or refractory DLBCL and FL. Further investigation is ongoing.

Keywords: Diffuse large B-cell lymphoma (DLBCL); follicular lymphoma (FL); histone deacetylase inhibitor (HDACi)

Submitted Oct 21, 2021. Accepted for publication Dec 15, 2021.

doi: 10.21037/atm-21-6019

View this article at: https://dx.doi.org/10.21037/atm-21-6019 


\section{Introduction}

Diffuse large B-cell lymphoma (DLBCL) is aggressive and genetically heterogeneous tumors, accounting for approximately $30 \%$ of non-Hodgkin's lymphoma (NHL) cases. Follicular lymphoma (FL) is the most prevalent indolent NHL (about $22 \%$ of cases) (1). Standard treatment with rituximab based chemo-immunotherapy has revolutionized the 5 years overall survival (OS) of DLBCL patients to $58 \%(2,3)$ and 10 years OS of FL to $80 \%$ (4). However, approximately $40 \%$ of DLBCL patients experiencing relapsed or refractory disease have a poor prognosis (5). Although FL has a median OS (mOS) more than 15 years, most patients with FL undergo disease progression after treatment. And 20\% of patients with FL progress or relapse in the first 2 years after first-line chemoimmunotherapy have significantly shorter survival (6). Patients with relapsed FL may also become refractory to chemoimmunotherapy or experience histological transformation to DLBCL. Prognosis remains poor for patients with relapsed or refractory DLBCL or FL (1). To date, multiple research have carried out to find the best treatment plan for these patients, so as to prolong the survival. In the PARMA trial, relapsed DLBCL patients showed significantly better event free survival (EFS) and OS in the salvage chemotherapy followed by high-dose therapy (HDT) and autologous stem-cell transplantation (ASCT) compared with the chemotherapy alone group (7). CUP trial has demonstrated that progression-free survival (PFS) and OS improved in relapsed FL patients treated with HDT followed by ASCT then those only received chemotherapy (8). For younger patients, HDT followed by ASCT can be taken into consideration (9). However, patients over 65 years old are usually diagnosed as an advanced stage with intermediate- to high-risk according to the International Prognostic Index (IPI) (10). Some of the elderly patients may have comorbidities, such as cardiopathy, diabetes and neuropathy, consider to be contraindications to some chemotherapeutics. Furthermore, most elderly patients do not qualify for intensive chemotherapy or not candidates for ASCT. Hence, effective and safe treatment is highly desirable for elderly patients with relapsed or refractory DLBCL and FL.

Rituximab is a chimeric type I anti-CD20 monoclonal antibody, significantly improved survival rates when added to chemotherapy in B cell lymphomas. The therapeutic effect of rituximab depends on the level of CD20 expression on the surface of malignant B cells. Aberrant down- modulation of CD20 expression was observed following rituximab treatment in some patients with B cell NHL $(11,12)$. The downregulation of the CD20 protein on tumor cells, could be one of the reasons for the decreased efficacy of anti-CD20-based therapeutic regimens $(13,14)$.

Chidamide, an oral benzamide class histone deacetylase inhibitors (HDACi) that selectively inhibits HDAC1, 2, 3, and 10 activity, was approved by the China Food and Drug Administration (CFDA) in 2015 for the treatment of relapsed or refractory peripheral $\mathrm{T}$ cell lymphoma. In recent studies, chidamide has also shown a potential benefit for patients with B cell lymphoma (15). In our previous study, we reported that a patient with relapsed/refractory DLBCL who received intensive pretreatment achieved a complete response (CR) after three-cycle regimens of chidamide plus rituximab (16). Moreover, chidamide plus rituximab, cyclophosphamide, doxorubicin, vincristine, and prednisone (R-CHOP) has demonstrated promising activity and manageable safety as a first-line treatment for elderly patients with DLBCL (17). So far we have confirmed that chidamide is a promising sensitizer for the retreatment of DLBCL with rituximab in vitro and in vivo. This further showed that the synergistic effect of chidamide on rituximab could potentially inhibited tumor growth and prolonged survival of relapsed or refractory DLBCL patients who are intolerable to R-CHOP or with poor response to rituximab (16).

However, prospective trials specifically investigating the efficacy of chidamide and rituximab in elderly patients with relapsed or refractory DLBCL or FL were sparse. Therefore, we aimed to assess the efficacy and safety of chidamide plus rituximab as a salvage regimen in elderly patients in China with relapsed or refractory DLBCL and FL.

We present the following article in accordance with the TREND reporting checklist (available at https://dx.doi. org/10.21037/atm-21-6019).

\section{Methods}

\section{Participants}

A multi-center, single-arm, open-label, phase II study was conducted at three hospitals in China (Tianjin Union Medical Center, Second Hospital of Dalian Medical University, and Shanxi Provincial Cancer Hospital). Eligible patients were 65 years or older, had an Eastern Cooperative Oncology Group (ECOG) performance status of 0-2 with an IPI $\geq 2$ and a life expectancy of more than 3 months. All patients were diagnosed with CD20-positive DLBCL and 
FL (histopathologically diagnosed) with either a relapsed or refractory history after one ( $\geq$ two cycles) or more lines of rituximab-associated regimens. Refractory was defined as patients not achieving partial or complete remission after two treatment cycles according to the Revised Response Criteria for Malignant Lymphoma (International Working Group 2007 criteria) (18). At least one measurable lesion of more than $15 \mathrm{~mm}$ in the longest diameter or more than $10 \mathrm{~mm}$ in the shortest diameter on enhanced CT scan or PET/CT was required for patients to be eligible. Patients' laboratory tests needed to conform to the following standards: hemoglobin $\geq 90 \mathrm{~g} / \mathrm{L}$, neutrophil count $\geq 1.5 \times 10^{9} / \mathrm{L}$, platelets $\geq 80 \times 10^{9} / \mathrm{L}$, alanine aminotransferase (ALT) or aspartate aminotransferase (AST) $\leq 2.5 \times$ upper limit of normal (ULN), alkaline phosphatase (AKP) or bilirubin $\leq 1.5 \times \mathrm{ULN}$, or creatinine $\leq 1.5 \times \mathrm{ULN}$ (unless the laboratory tests abnormalities were caused by lymphoma).

Patients were excluded from the study if they had central nervous system (CNS) lymphoma or testicular lymphoma; a history of any other malignant tumor; ASCT; previous exposure to any HDACi; uncontrollable cardiovascular, autoimmune, coagulation, or infectious disease or hepatic or renal insufficiency; exposure to any investigational agent within 4 weeks of the first dose of the study drug; exposure to the last dose of radiotherapy or antitumor treatment (chemotherapy, targeted therapy, immunotherapy, or arterial embolization) within 3 weeks of the first dose of the study drug; inability to comply with the protocol for mental or other unknown reasons; pregnancy or lactation; positive for hepatitis B virus (HBV-DNA) or human immunodeficiency virus (HIV). The study protocol described the complete inclusion and exclusion criteria, and has been registered in the Chinese Clinical Trial Registry (Identifier: ChiCTR2100050169). The study was conducted in accordance with the Declaration of Helsinki (as revised in 2013). The final protocol, amendments, patient informed consent, and any other appropriate documents were reviewed and approved by the Ethics Committee of Tianjin Union Medical Center (approval number 2018-9). Written informed consent was obtained from all patients before enrollment in the study.

\section{Interventions}

Patients received chidamide (Epidaza ${ }^{\circledR}$, China) $10 \mathrm{mg}$ orally on days 1-6 and 8-14 either at home or hospital. According to our previous research (18), rituximab (Hanlikang ${ }^{\circledR}$, China) $375 \mathrm{mg} / \mathrm{m}^{2}$ was given intravenously at the hospital by nursing staff on day 7 , then once every 3 weeks until disease progression, death, unacceptable toxicity, or withdrawal of consent, for a maximum of 24 months. The chidamide provided to subjects was postponed if any adverse events (AEs) $\geq$ grade 3 occurred. There was no dose modification plan for chidamide. The regimen resumed when the AEs resolved to grade $0-1$ or baseline level. Treatment was permanently terminated for patients who had stopped chidamide for more than 6 weeks. Granulocyte colonystimulating factor (G-CSF) and thrombopoietin (TPO) were given if grade $\geq 3$ neutropenia or thrombocytopenia were present.

Basic information was collected before treatment, including age, gender, weight, IPI score or FLIPI-2, Ann Arbor staging, accompanying B symptoms, ECOG, and previous regimens and cycles. Baseline evaluations were performed in the week preceding the first dose and included a physical examination, complete blood cell count, coagulation function, serum biochemistry with lactate dehydrogenase (LDH) levels, $\beta$-actin, HBV markers and DNA, Epstein-Barr virus DNA (EBV DNA), and an electrocardiogram. Bone marrow aspiration and trephine biopsy, PET-CT or enhanced CT scan (including cranial, neck, chest, abdomen, pelvis) were performed 28 days before treatment.

Treatment response was assessed by PET-CT or enhanced CT scan every two cycles according to the Lugano classification 2014 (19). Patients who achieved a complete or partial response (PR) or were in a stable condition received another two cycles. Patients stopped receiving the regimen if the disease progressed. Safety was assessed by laboratory tests, electrocardiography, and vital sign monitoring at each cycle, and all adverse events were recorded.

\section{Objectives and outcomes}

The primary endpoint was the objective response rate (ORR) assessed by PET-CT or enhanced CT scan. The secondary endpoints were the disease control rate (DCR), PFS, OS, and AEs. PFS was measured from the first cycle of the current treatment to the date of progression, relapse, or death from any cause. OS was recorded from the first cycle of the current treatment to death from any cause or the date of the last follow-up. Safety was evaluated in patients who received at least one dose of the therapy. AEs were assessed according to the National Cancer Institute Common Terminology Criteria for Adverse Events (CTCAE, version 


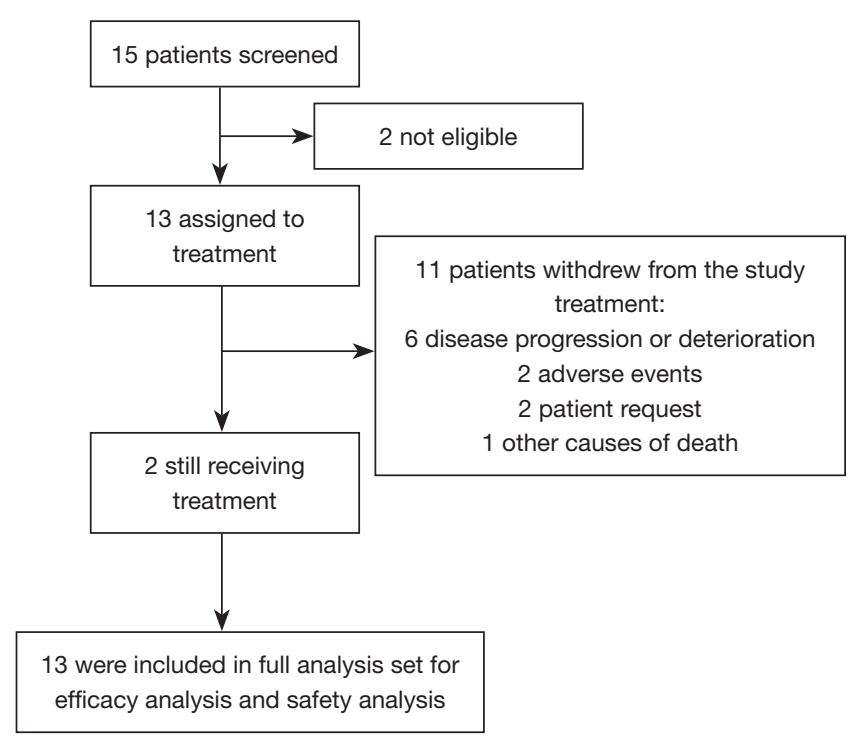

Figure 1 Trial profile. Cutoff date June 20, 2021.

4.03). Treatment-related adverse events (TRAEs) were defined as those that were related, or possibly related, to treatment or whose relationship to treatment was uncertain, as assessed by the investigator. AEs were collected by face-to-face interviewer-administered questionnaires, and survival information was gathered by telephone follow-up.

\section{Statistical analysis}

For this phase II single-arm study, analyses were performed at the individual level. Sample size estimation was performed by Fleming's single-stage procedure. Defining p0 as the proportion of responses below which the treatment did not warrant further investigation and $\mathrm{p} \alpha$ as the proportion of responses beyond which a phase 3 trial should be carried out, we set $\mathrm{P}_{0}=0.05$ and $\mathrm{P}_{\alpha}=0.35$. Given a significance level of $5 \%$ and power of $80 \%$, the number of patients required was 12 , and the number of successful patients was 10 . The number of patients was set at 13 to take into account an estimated dropout rate of $10 \%$. Statistical analyses were performed by GraphPad Prism 6 (Version 6.01). Survival estimates were calculated by the Kaplan-Meier method, and survival curves were compared by the log-rank test. A two-sided $\mathrm{P}$ value of $<0.05$ was considered statistically significant.

\section{Results}

\section{Patient characteristics}

A total of 13 patients with relapsed/refractory DLBCL or FL were enrolled and treated between November 2018 and December 2020 at the three study sites (Tianjin Union Medical Center, China; The Second Hospital of Dalian Medical University, China; Sanxi Provincial Cancer Hospital, China) (Figure 1). The DLBCL cohort comprised ten patients, while the FL cohort included three patients. The baseline characteristics of the patients are summarized in Table 1. The median age was 72.5 years (65-79 years) and 65 years (65-75 years) for the DLBCL and FL cohorts, respectively. Most patients were female (84.6\%) with an ECOG performance status score of $0-1$. A large proportion of patients in the DLBCL cohort $(80 \%)$ presented with advanced lymphoma that had progressed from at least one regimen (median 2, 1-6). All patients in the FL cohort (100\%) had stage III or IV disease and had received significant prior systemic cancer therapy with a median number of 6 [1-9] prior regimens. The median number of previous antineoplastic treatments cycles was 10 [2-23] in the DLBCL group and 19 [4-65] in the FL group. All enrolled patients received R-CHOP or rituximab, cyclophosphamide, vincristine, and prednisolone (R-CVP) at the first-line setting. Additionally, $90 \%$ of subjects with DLBCL and $100 \%$ with FL had at least one extra-nodal involvement. Four subjects showed elevated LDH levels.

By the data cutoff date of June 20, 2021, the median follow-up was 13.4 months (5.9-31.2 months). The median cycles patients received were 2 (1-12 cycles). Two patients continued to receive the study treatment. The reasons for treatment discontinuation in the other patients were disease progression $(n=6)$, unbearable $\mathrm{AE}(\mathrm{n}=2)$, patient request $(n=2)$, and non-tumor-related death $(n=1)$.

\section{Efficacy}

All 13 patients were included in the efficacy analysis, and tumor evaluations are listed in Table 2. ORR and DCR were achieved in $40 \%$ of the ten patients with DLBCL, with three patients $(30.0 \%)$ achieving a CR and one patient $(10.0 \%)$ achieving a PR. The median time to initial response was 2.1 months (1.4-3.3 months) 
Table 1 Baseline clinical and pathological characteristics

\begin{tabular}{|c|c|c|}
\hline Characteristics & DLBCL $(n=10)$ & $\mathrm{FL}(\mathrm{n}=3)$ \\
\hline \multicolumn{3}{|l|}{ Age (years) } \\
\hline Median [range] & 72.5 [65-79] & 65.0 [65-75] \\
\hline \multicolumn{3}{|l|}{ Gender } \\
\hline Male & $2(20.0 \%)$ & - \\
\hline Female & 8 (80.0\%) & $3(100.0 \%)$ \\
\hline \multicolumn{3}{|l|}{ ECOG } \\
\hline 0 & 9 (90.0\%) & $2(66.7 \%)$ \\
\hline 1 & $1(10.0 \%)$ & $1(33.3 \%)$ \\
\hline \multicolumn{3}{|l|}{ Ann arbor stage } \\
\hline II & 2 (20.0\%) & - \\
\hline III & $2(20.0 \%)$ & - \\
\hline IV & $6(60.0 \%)$ & 3 (100.0\%) \\
\hline \multicolumn{3}{|l|}{ LDH } \\
\hline Normal & 7 (70.0\%) & $2(66.7 \%)$ \\
\hline Elevated & 3 (30.0\%) & $1(33.3 \%)$ \\
\hline \multicolumn{3}{|l|}{ Extranodal sites } \\
\hline 0 & $1(10.0 \%)$ & - \\
\hline$\geq 1$ & 9 (90.0\%) & $3(100.0 \%)$ \\
\hline \multicolumn{3}{|l|}{ IPI/FLIPI-2 } \\
\hline 2 & $3(30.0 \%)$ & $1(33.3 \%)$ \\
\hline 3 & 3 (30.0\%) & $1(33.3 \%)$ \\
\hline 4 & $4(40.0 \%)$ & $1(33.3 \%)$ \\
\hline $\begin{array}{l}\text { Cycles of prior systemic } \\
\text { therapy, median [range] }\end{array}$ & 10 [2-23] & 19 [4-65] \\
\hline $\begin{array}{l}\text { Lines of prior systemic } \\
\text { therapy, median [range] }\end{array}$ & $2[1-6]$ & $6[1-9]$ \\
\hline Prior rituximab & 10 (100.0\%) & $3(100.0 \%)$ \\
\hline
\end{tabular}

DLBCL, diffuse large B cell lymphoma; FL, follicular lymphoma; ECOG, Eastern Cooperative Oncology Group; LDH, lactate dehydrogenase; IPI, International Prognostic Index (for DLBCL); FLIPI-2, Follicular lymphoma International Prognostic Index (for FL).

in the four patients with an objective response, and the median duration of response (DoR) was not reached (2.2-29.7 months). In the FL cohort, ORR and DCR were achieved in $100 \%$ of patients, one patient achieved a CR (33.3\%), and two patients achieved a PR (66.7\%).
The median time to initial response was 1.5 months (1.4-2.3 months) in the three patients with an objective response, with $\mathrm{mDoR}$ not reached (4.4-12.1 months).

Overall clinical benefit was observed in $53.8 \%$ of the evaluable patients. The maximum percentage reductions from baseline in the target lesions of patients with available data are shown in Figure $2 A$ and responses during treatment are shown in Figure $2 B$.

\section{Survival outcome}

We further analyzed the survival outcome of the cohort. The PFS and OS from the start of chidamide plus rituximab treatment were evaluated. Based on the Kaplan-Meier estimation, the median PFS (mPFS) of the DLBCL cohort was 2.6 months (0.9-31.2 months, Figure $3 A)$, whereas the mOS was 16.7 months (3.3-31.2 months, Figure $3 B$ ) at the time of data cutoff. Of the four DLBCL subjects with an objective response, all remained progression free for $\geq 90$ days. The FL cohort did not reach the mPFS (6.6-13.6 months, Figure 3A) or the mOS (6.6-13.6 months, Figure $3 B$ ). For all patients, the mPFS was 6.8 months, and the mOS was 16.7 months (Figure $3 C, 3 D$ ).

\section{Safety}

Safety analyses were based on the 13 eligible patients. The median number of treatment doses with chidamide and rituximab was 2 (1-10 doses). The overall incidence of drug-related AEs was similar in the DLBCL and FL cohorts. Treatment-related AEs occurred in nine (69.2\%) patients. The most common treatment-related AEs were leukopenia $(38.5 \%)$, neutropenia $(30.8 \%)$, lymphopenia (30.8\%), thrombocytopenia (30.8\%), fatigue (38.5\%), and hyperuricemia $(30.8 \%)$. The incidence of grade 3-4 treatment-related AEs was $46.2 \%$. The most common were leukopenia (15.4\%), lymphocytopenia (15.4\%), and thrombocytopenia (15.4\%) (Table 3). Treatment-related AEs did not lead to dose reduction. Treatment-related serious AEs were reported in two patients (15.4\%), including one case of grade 4 hypothrombocytopenia along with grade 4 hyperuricemia, and one case of grade 4 fatigue. All grade 3-4 treatment-related AEs were resolved by symptomatic treatment in addition to supportive care, with most of them recovering below grade 1 . Treatment-related AEs resulting in discontinuation occurred in two patients with fatigue and slight interstitial lung diseases, respectively. There were no 
Table 2 Activity of chidamide plus rituximab in elderly patients with relapse and refractory B cell lymphoma

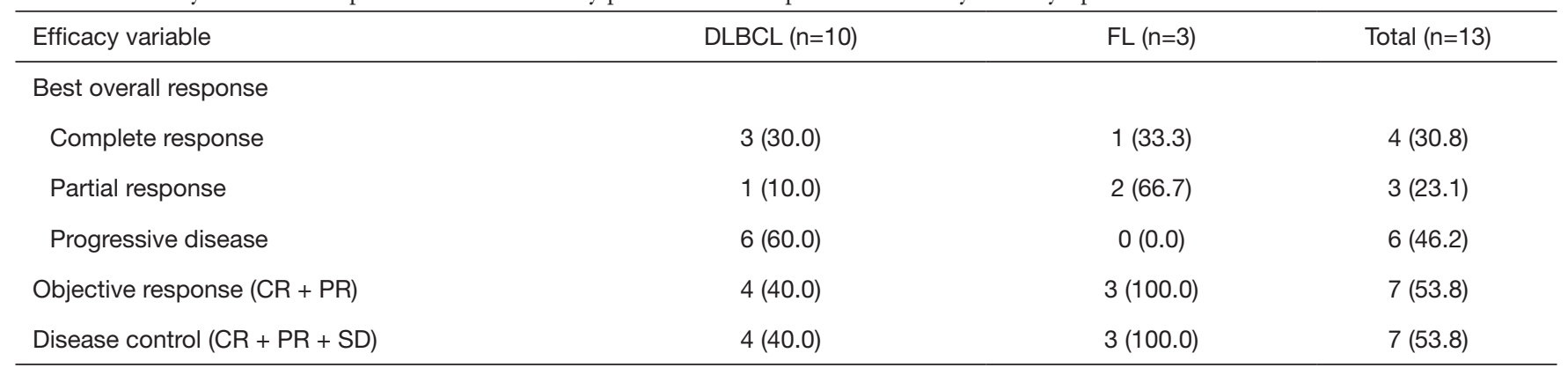

Data presented as [cases (\%)]. DLBCL, diffuse large B cell lymphoma; FL, follicular lymphoma; CR, complete response; PR, partial response; SD, stable disease.
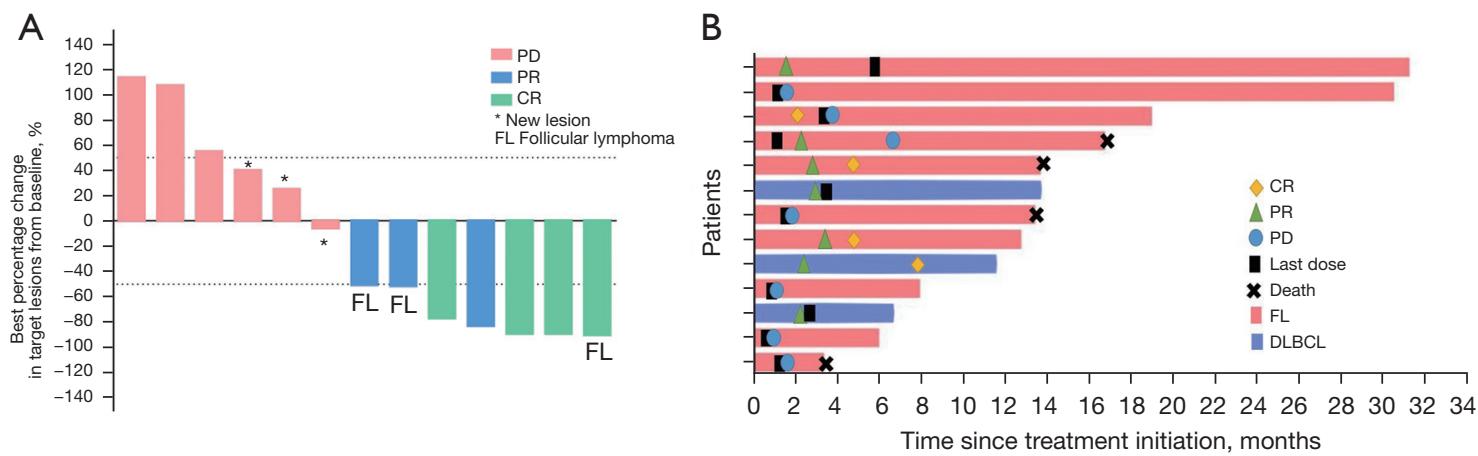

Figure 2 Overall tumor responses of chidamide plus rituximab as assessed by site investigators. (A) The best change from baseline in the size of the target tumor lesion. The color code defines the best response of the target tumor lesion. Three patients (indicated by stars) with new lesions are evaluated for disease progression. (B) Records of responses during treatment. The length of each bar represents the time from treatment initiation to the last follow-up. CR, complete response; PR, partial response; SD, stable disease; PD, progressive disease; FL, follicular lymphoma; DLBCL, diffuse large B cell lymphoma.

treatment-related deaths.

\section{Discussion}

Elderly patients with relapsed or refractory DLBCL and FL have poor clinical outcomes and limited treatment options. In this multicenter, single-arm, phase II study, chidamide and rituximab demonstrated promising antitumor activity and manageable toxic effects in heavily pretreated elderly patients with relapsed or refractory DLBCL and FL. Moreover, the regimen significantly shortened the length of hospital stay and improved compliance rates of elderly patients. To our knowledge, this is the first report to evaluate the efficacy and safety of combined chidamide and rituximab as a second-line and subsequent-line regimen for elderly patients with relapsed or refractory B-cell lymphoma.
In our study, the median age was 71 years (65-79 years) years in all B cell lymphoma patient. The prognosis of recruited patients in our trial presented with an IPI/ FLIPI-2 score above 3 (70\% of DLBCL, $66.7 \%$ of FL). The results of our study were reflected in both primary and secondary endpoints, with the best ORR of $40 \%$ and $100 \%$ for elderly patients with relapsed or refractory DLBCL and FL, respectively. Comparing with an Italian retrospective study on relapsed or refractory DLBCL patients treated with rituximab plus bendamustine (BR), our cohort had a survival benefit of approximately 6 months in mOS (10.8 vs. 16.7 months) (20). And patients had a higher rate of intermediate-high or high-risk IPI in our research $(56 \%$ vs. $70 \%)$. The results were encouraging, although no benefit was observed in ORR (50\% vs. $40 \%)$ and mPFS (2.8 vs. $8.8 \mathrm{~m}$ ) in our study. In another study, only $41 \%$ of relapsed or refractory indolent NHL (including FL) of all age 
A

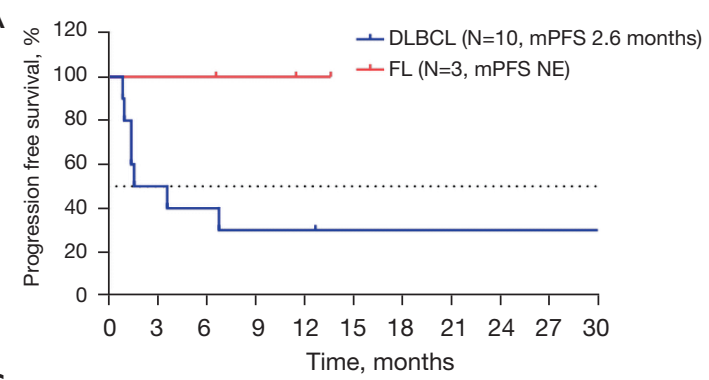

C

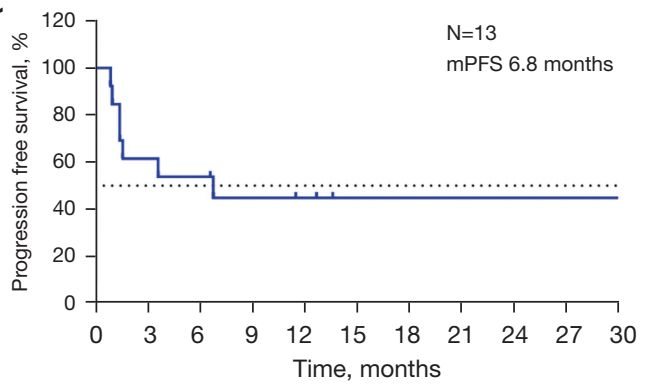

B

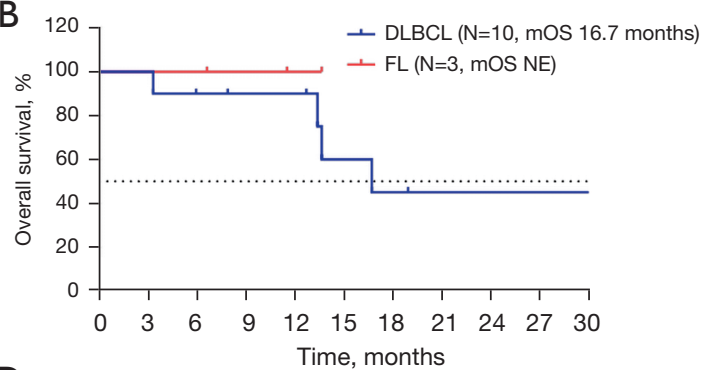

D

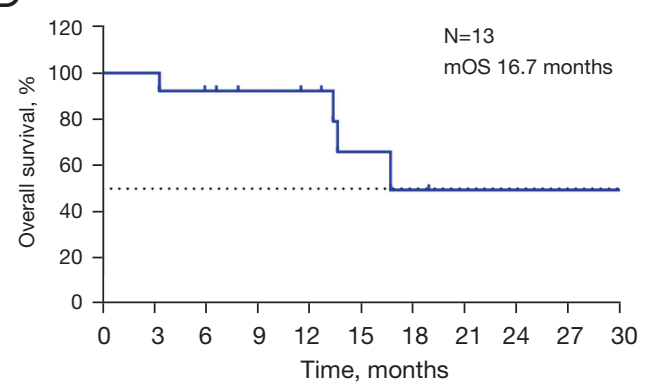

Figure 3 Kaplan-Meier estimation of the survival of patients with relapsed or refractory B cell lymphoma treated with chidamide and rituximab. The analyses for PFS (A) and OS (B) of chidamide combined with rituximab-treated patients with DLBCL (blue line) and FL (red line) expressed in months. PFS (C) and OS (D) curves for all patients in the efficacy evaluation. The dotted line indicates the median survival. DLBCL, diffuse large B cell lymphoma; FL, follicular lymphoma; PFS, progression-free survival; OS, overall survival; NE, not estimable.

(44-85 years) response to vorinostat and rituximab. While all 3 patients with FL in our study had better response (100\%) to HDACi based regimen and AEs were similar to Chen et al.'s study (21). Thus, our findings confirm that chidamide and rituximab have the potential to improve outcomes in elderly patients with relapsed or refractory DLBCL and FL.

This study addressed the issue of whether the rituximab would continue to be active for retreatment after disease progression. Our previous study suggested that the downregulation of CD20 mRNA in DLBCL cell lines is caused by HDAC-mediated gene silencing (16). Internalization of the CD20 molecule (22) and loss of the CD20/rituximab complex from the cell surface (23) also contribute to rituximab-induced downregulation of CD20 expression. The downregulation of the $\mathrm{CD} 20$ protein on tumor cells, may affects the rituximab-induced lipid raft domain organization and downstream signal interference, result in decreased efficacy of anti-CD20-based therapeutic regimens $(13,14)$. To date, several mechanisms associated with resensitizing of CD20 monoclonal antibody by HDAC inhibition have been identified. Bobrowicz et al.'s study (24) had demonstrated that the inhibition of HDAC6 could upregulates the protein levels of CD20 independently. Xue et al. (25) confirmed that vorinostat overcame acquired resistance to rituximab- and chemo-therapy in aggressive B cell lymphoma by increasing p21 and acetylation of histone H3 leading to G1 cell cycle arrest, which could activate alternative cell death pathways. Previously, our research has illustrated that chidamide can decreased that of phophoHDAC3 and upregulate the expression of CD20 (16). As a result, the sensitivity of tumor cells to CD20 monoclonal antibody is restored by maintain sufficient targeting levels. These findings, suggest that HDACis are a reasonable therapeutic strategy implemented in combination therapies with anti-CD20 monoclonal antibodies.

The response of patients was different in this trial. One patient had a CR after two cycles of treatment, and six patients had PR after the initial two cycles, three of them improved to CR within 6 cycles (3-6 cycles). Responses were generally durable in both cohorts, the mDoR lasting more than 90 days in all responders. However, PD was observed in five DLBCL patients (Figure 1B). Recent study has confirmed that inactivating mutations of the histone acetyltransferase CREBBP (26), exist in 6.4-22.3\% of DLBCL patients and $30.8-68 \%$ of FLs (27-29), is correlated with chidamide sensitivity in B cell lymphoma 
Table 3 Treatment-related adverse events observed with chidamide and rituximab

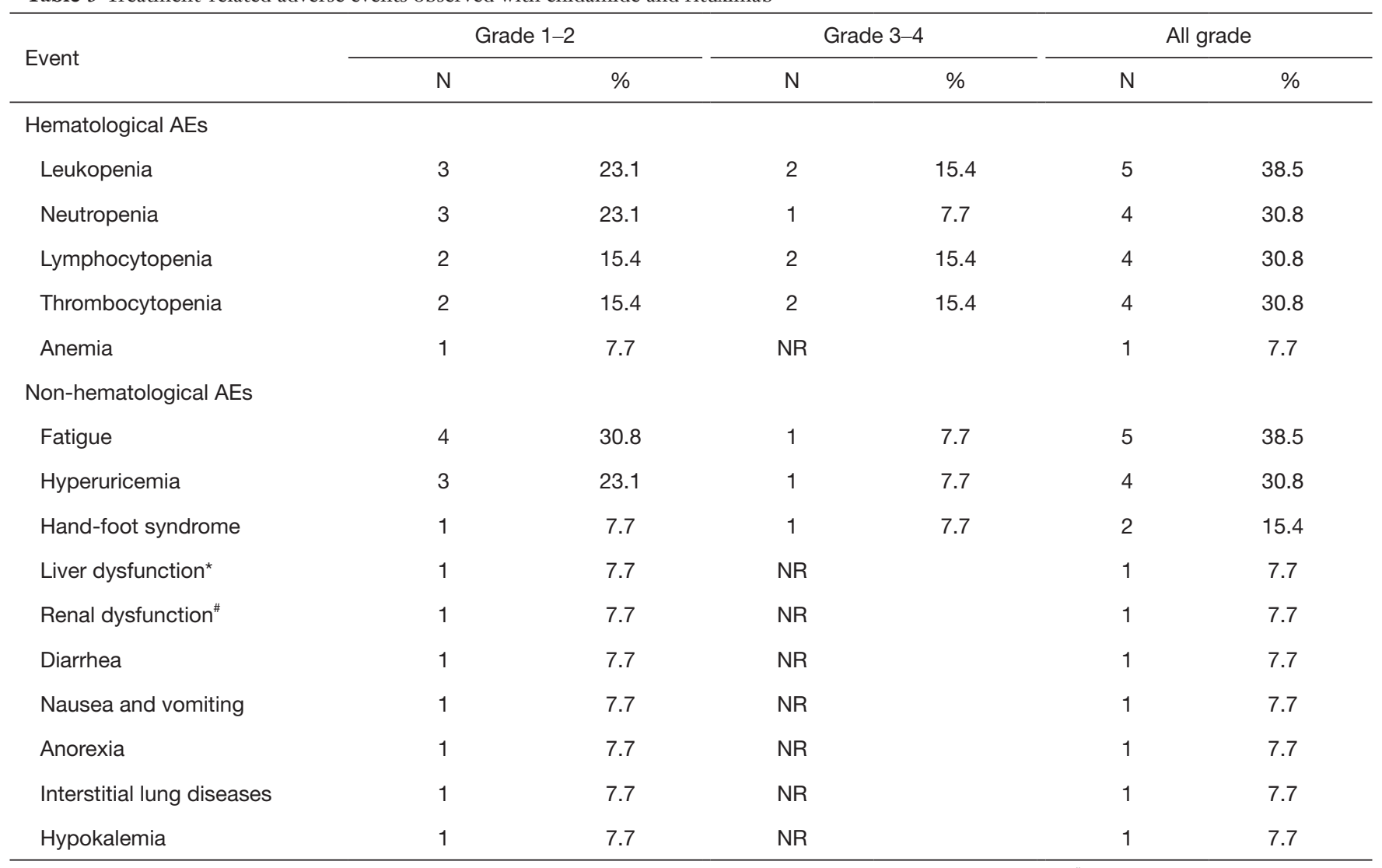

*, liver dysfunction: elevated serum aspartate aminotransferase levels and alanine aminotransferase levels; ", renal dysfunction: elevated serum creatinine and urea. AE, advance events; NR, not reported.

patients. The loss of CREBBP lead to unopposed deacetylation of specific genes by the BCL6/SMRT/ HDAC3 complex (27). Although no significant difference in survival between patients with or without CREBBP mutations upon treatment with CR-CHOP in DLBCL patients, patients with inactivation mutation of CREBBP showed an inferior PFS and OS (17). Therefore, CREBBP can serve as a potential biomarker to predict chidamide sensitivity (30), and patients with CREBBP mutation are likely to benefit from our regimen.

Regarding the dose intensity and feasibility of the chidamide and rituximab regimen in elderly patients with relapsed or refractory DLBCL or FL, the analysis of drug delivery showed that patients received full doses of both drugs in all cycles which demonstrated a better tolerability. The standard protocol of chidamide for the treatment of T-cell lymphoma is $30 \mathrm{mg}$, twice a week. In the phase I trial of chidamide, no dose-limiting toxicity were identified in the BIW cohorts up to $50 \mathrm{mg}$ (31). Considering the older age of B cell NHL patients and less tolerate to the drug, we reduced dose of chidamide to $10 \mathrm{mg} /$ day for 2 weeks (except seventh day) with rituximab on the seventh day, then rest for a week. For patients under 65 years old, maintain $10 \mathrm{mg} /$ day or modify the dose of chidamide could be taken into consideration, but further investigations are needed.

Although chidamide was given in a novel way, AEs which associated were generally mild and manageable with no additional AE. The main AEs were myelosuppression, fatigue, and hyperuricemia. The most common grade $3 / 4$ adverse event was myelosuppression $(\leq 15.4 \%)$, which is similar to, or lower than, other previously reported HDACiassociated regimens $(9,20,21,31)$. The myelosuppression was of short duration and resolved using G-CSF without translating into excessive infections. Two patients discontinued the therapy because of intolerant fatigue and light interstitial lung diseases, respectively. Both patients 
recovered in one week after stop the treatment. There was no treatment-related death. No unexpected AEs or new safety signals were noted with the addition of chidamide to rituximab.

The limitations of the present study included the singlearm design and the relatively small sample size. Therefore, we were unable to compare our findings directly with the treatment outcomes of chemotherapy alone. Furthermore, other biomarkers, including EB virus positivity, were not detected in our trial.

In conclusion, our results confirmed that chidamide combined with rituximab in elderly patients over 65 years with relapsed or refractory B cell lymphoma may be a potential second-line or follow-up treatment option with a manageable safety profile and promising efficacy. A randomized phase III trial of chidamide plus rituximab is warranted to further evaluate the combination as a secondline or follow-up treatment for relapsed or refractory DLBCL and FL at all ages in the near future.

\section{Acknowledgments}

We thank Jiangsu Fosun Pharmaceutical Sales Co. Ltd. (Xuzhou, Jiangsu, China). We also thank all the patients, their families, and the site investigators who participated in the study.

Funding: This study was funded by the National Science and Technology Major Project of China (82070206).

\section{Footnote}

Reporting Checklist: The authors have completed the TREND reporting checklist. Available at https://dx.doi. org/10.21037/atm-21-6019

Data Sharing Statement: Available at https://dx.doi. org/10.21037/atm-21-6019

Conflicts of Interest: All authors have completed the ICMJE uniform disclosure form (available at https://dx.doi. org/10.21037/atm-21-6019). The authors report that the research was conducted in the absence of any commercial or financial relationships with Jiangsu Fosun Pharmaceutical Sales Co. Ltd. The authors have no other conflicts of interest to declare.

Ethical Statement: The authors are accountable for all aspects of the work in ensuring that questions related to the accuracy or integrity of any part of the work are appropriately investigated and resolved. The final protocol, amendments, informed consent, and any other appropriate documents were reviewed and approved by the Ethics Committee of Tianjin Union Medical Center (Approval Number 2018-9). The study was conducted in accordance with the principles of the Declaration of Helsinki (as revised in 2013). Written informed consent was obtained from all patients before enrollment in the study.

Open Access Statement: This is an Open Access article distributed in accordance with the Creative Commons Attribution-NonCommercial-NoDerivs 4.0 International License (CC BY-NC-ND 4.0), which permits the noncommercial replication and distribution of the article with the strict proviso that no changes or edits are made and the original work is properly cited (including links to both the formal publication through the relevant DOI and the license). See: https://creativecommons.org/licenses/by-nc-nd/4.0/.

\section{References}

1. Timmerman J, Herbaux C, Ribrag V, et al. Urelumab alone or in combination with rituximab in patients with relapsed or refractory B-cell lymphoma. Am J Hematol 2020;95:510-20.

2. Schuster SJ, Bishop MR, Tam CS, et al. Tisagenlecleucel in Adult Relapsed or Refractory Diffuse Large B-Cell Lymphoma. N Engl J Med 2019;380:45-56.

3. Feugier P, Van Hoof A, Sebban C, et al. Long-term results of the R-CHOP study in the treatment of elderly patients with diffuse large B-cell lymphoma: a study by the Groupe d'Etude des Lymphomes de l'Adulte. J Clin Oncol 2005;23:4117-26.

4. Freedman A, Jacobsen E. Follicular lymphoma: 2020 update on diagnosis and management. Am J Hematol 2020;95:316-27.

5. Wang SB, Chen JY, Zhao WL, et al. Efficacy of salvage radiotherapy for relapsed/refractory diffuse large B-cell lymphoma. Transl Cancer Res 2019;8:1019-26.

6. Carbone A, Roulland S, Gloghini A, et al. Follicular lymphoma. Nat Rev Dis Primers 2019;5:83.

7. Philip T, Guglielmi C, Hagenbeek A, et al. Autologous bone marrow transplantation as compared with salvage chemotherapy in relapses of chemotherapysensitive non-Hodgkin's lymphoma. N Engl J Med 1995;333:1540-5.

8. Schouten HC, Qian W, Kvaloy S, et al. High-dose 
therapy improves progression-free survival and survival in relapsed follicular non-Hodgkin's lymphoma: results from the randomized European CUP trial. J Clin Oncol 2003;21:3918-27.

9. Straus DJ, Hamlin PA, Matasar MJ, et al. Phase I/II trial of vorinostat with rituximab, cyclophosphamide, etoposide and prednisone as palliative treatment for elderly patients with relapsed or refractory diffuse large B-cell lymphoma not eligible for autologous stem cell transplantation. Br J Haematol 2015;168:663-70.

10. Pfreundschuh $M$, Schubert J, Ziepert $M$, et al. Six versus eight cycles of bi-weekly CHOP-14 with or without rituximab in elderly patients with aggressive CD20+ B-cell lymphomas: a randomised controlled trial (RICOVER-60). Lancet Oncol 2008;9:105-16.

11. Miyoshi H, Arakawa F, Sato K, et al. Comparison of CD20 expression in B-cell lymphoma between newly diagnosed, untreated cases and those after rituximab treatment. Cancer Sci 2012;103:1567-73.

12. Hiraga J, Tomita A, Sugimoto T, et al. Down-regulation of CD20 expression in B-cell lymphoma cells after treatment with rituximab-containing combination chemotherapies: its prevalence and clinical significance. Blood 2009; 113:4885-93.

13. Shimizu R, Kikuchi J, Wada T, et al. HDAC inhibitors augment cytotoxic activity of rituximab by upregulating CD20 expression on lymphoma cells. Leukemia 2010;24:1760-8.

14. Nowakowski GS, Blum KA, Kahl BS, et al. Beyond RCHOP: A Blueprint for Diffuse Large B Cell Lymphoma Research. J Natl Cancer Inst 2016;108:djw257.

15. Li Q, Huang J, Ou Y, et al. Progressive diffuse large B-cell lymphoma with TP53 gene mutation treated with chidamide-based chemotherapy. Immunotherapy 2019;11:265-72.

16. Guan XW, Wang HQ, Ban WW, et al. Novel HDAC inhibitor Chidamide synergizes with Rituximab to inhibit diffuse large B-cell lymphoma tumour growth by upregulating CD20. Cell Death Dis 2020;11:20.

17. Zhang MC, Fang Y, Wang L, et al. Clinical efficacy and molecular biomarkers in a phase II study of tucidinostat plus R-CHOP in elderly patients with newly diagnosed diffuse large B-cell lymphoma. Clin Epigenetics 2020;12:160.

18. Cheson BD, Pfistner B, Juweid ME, et al. Revised response criteria for malignant lymphoma. J Clin Oncol 2007;25:579-86.

19. Cheson BD, Fisher RI, Barrington SF, et al.
Recommendations for initial evaluation, staging, and response assessment of Hodgkin and non-Hodgkin lymphoma: the Lugano classification. J Clin Oncol 2014;32:3059-68.

20. Arcari A, Chiappella A, Spina M, et al. Safety and efficacy of rituximab plus bendamustine in relapsed or refractory diffuse large B-cell lymphoma patients: an Italian retrospective multicenter study. Leuk Lymphoma 2016;57:1823-30.

21. Chen R, Frankel P, Popplewell L, et al. A phase II study of vorinostat and rituximab for treatment of newly diagnosed and relapsed/refractory indolent non-Hodgkin lymphoma. Haematologica 2015;100:357-62.

22. Beers SA, French RR, Chan HT, et al. Antigenic modulation limits the efficacy of anti-CD20 antibodies: implications for antibody selection. Blood 2010;115:5191-201.

23. Williams ME, Densmore JJ, Pawluczkowycz AW, et al. Thrice-weekly low-dose rituximab decreases CD20 loss via shaving and promotes enhanced targeting in chronic lymphocytic leukemia. J Immunol 2006;177:7435-43.

24. Bobrowicz M, Dwojak M, Pyrzynska B, et al. HDAC6 inhibition upregulates CD20 levels and increases the efficacy of anti-CD20 monoclonal antibodies. Blood 2017;130:1628-38.

25. Xue K, Gu JJ, Zhang Q, et al. Vorinostat, a histone deacetylase (HDAC) inhibitor, promotes cell cycle arrest and re-sensitizes rituximab- and chemo-resistant lymphoma cells to chemotherapy agents. J Cancer Res Clin Oncol 2016;142:379-87.

26. Lunning MA, Green MR. Mutation of chromatin modifiers; an emerging hallmark of germinal center B-cell lymphomas. Blood Cancer J 2015;5:e361.

27. Jiang Y, Ortega-Molina A, Geng H, et al. CREBBP Inactivation Promotes the Development of HDAC3Dependent Lymphomas. Cancer Discov 2017;7:38-53.

28. Rosenwald A, Wright G, Chan WC, et al. The use of molecular profiling to predict survival after chemotherapy for diffuse large-B-cell lymphoma. N Engl J Med 2002;346:1937-47.

29. Xu PP, Fu D, Li JY, et al. Anthracycline dose optimisation in patients with diffuse large B-cell lymphoma: a multicentre, phase 3, randomised, controlled trial. Lancet Haematol 2019;6:e328-37.

30. Sun Y, Gao Y, Chen J, et al. CREBBP cooperates with the cell cycle machinery to attenuate chidamide sensitivity in relapsed/refractory diffuse large B-cell lymphoma. Cancer Lett 2021;521:268-80. 
31. Dong M, Ning ZQ, Xing PY, et al. Phase I study of chidamide (CS055/HBI-8000), a new histone deacetylase inhibitor, in patients with advanced solid tumors and lymphomas. Cancer Chemother Pharmacol 2012;69:1413-22.

Cite this article as: Chen $X$, Wang $H$, Sun $X, S u L$, Liu F, Zhao K, Xu L, Wu S, Song T. Safety of chidamide plus rituximab in elderly patients with relapsed or refractory B-cell lymphoma in China: a multicenter, single-arm, phase II study. Ann Transl Med 2021;9(24):1769. doi: 10.21037/atm-21-6019 